\title{
La respuesta tisular a implantes dentales con plataforma reducida (platform switching)
}

\section{Tissue response to dental implants with platform switching}

M.J. Bish González*, I. Ortiz Garcia**, A. Jiménez Guerra**, L. Monsalve Guil**, J. Moreno Muñoz***, E. Núñez Márquez***, E. Velasco Ortega****

\section{RESUMEN}

La evolución en los diseños de los sistemas de implantes y en la configuración de los pilares protésicos ha desarrollado el concepto de plataforma reducida que comprende la colocación de un pilar más estrecho que la plataforma del implante para aumentar su distancia de la interfase hueso-implante. La plataforma reducida es considerada un factor importante para preservar la estabilidad del hueso crestal y de los tejidos blandos y asegurar el éxito de los implantes dentales a largo plazo. La plataforma reducida reduce las fuerzas oclusales y la contaminación bacteriana en la interfase entre el hueso crestal y el implante. Los estudios experimentales en animales y clínicos en pacientes muestran su eficacia para prevenir la pérdida ósea y de los tejidos blandos periimplantarios

Conclusiones. La introducción de la plataforma reducida constituye un importante campo en la investigación experimental en implantología oral y en el tratamiento clínico con implantes para mantener los tejidos periimplantarios.

PALABRAS CLAVE: Implantes dentales, plataforma del implante, plataforma reducida, superficie de implante, superficie rugosa, oseointegración.

\section{ABSTRACT}

The evolution of designs of implant systems and abutments configurations has developed the concept of platform-switching that involves the connection of a narrower abutment to the platform implant to allow horizontal distance of the interface bone-implant. Platform-switching is considered an important factor to preserve the stability of crestal bone and soft tissue ensuring the success of dental implants in the long-term follow-up. Platform-switching reduces the forces of occlusal loading and bacterial contamination in the interface between the crestal bone and the implant. Experimental studies in animals and clinical studies in patients showed that implants with platform-switching have demonstrated the effectiveness to prevent peri-implant bone loss and subsequent soft tissue loss.

Conclusions. The platform-switching constitute an important field for research of experimental implant dentistry and clinical implant treatment in the maintenance of peri-implant tissues.

KEY WORDS: Dental implants, platform implant, platform switching, surface implant, rough surface, osseointegration.

* $\quad$ Licenciada en Odontología. Máster de Implantologia Oral. Facultad de Odontología. Universidad de Sevilla.

** Profesor Asociado de Odontología Integrada de Adultos y Gerodontología. Profesor del Máster de Implantología Oral. Facultad de Odontología. Universidad de Sevilla.

*** Profesor Asistente Honorario de Odontología Integrada de Adultos. Y Gerodontologia. Profesor del Máster de Implantología Oral.Facultad de Odontología. Universidad de Sevilla.

**** Profesor Titular de Odontología Integrada de Adultos. Director del Máster de Implantología Oral. Facultad de Odontología. Universidad de Sevilla. 
Fecha de recepción: 16 de marzo de 2020

Fecha de aceptación: 27 de marzo de 2020

M.J. Bish González, I. Ortiz Garcia, A. Jiménez Guerra, L. Monsalve Guil, J. Moreno Muñoz, E. Núñez Márquez, E. Velasco Ortega. La respuesta tisular a implantes dentales con plataforma reducida (platform switching). 2020; 36, (2): 107-115.

\section{INTRODUCCIÓN}

Los implantes dentales se han convertido en un método eficaz para la sustitución de los dientes perdidos y ha revolucionado la rehabilitación prostodónccia de los pacientes parcial y totalmente edéntulos ${ }^{1-2}$. La interfase hueso-implante juega un papel importante en conseguir la oseointegración y prolongar la supervivencia y la efectividad de los implantes. El grado y la calidad de la oseointegración depende de una serie de factores, que afectan significativamente a la respuesta inicial de las células óseas sobre el implante y a la estabilidad primaria después de la cirugía ${ }^{3-4}$.

La superficie de los implantes constituye un aspecto importante de la implantología oral porque sus características físicas y químicas pueden influir en el desarrollo de una mejor respuesta tisular del huésped a los implantes ${ }^{5-6}$. En este sentido, la energía, carga y composición de la superficie de los implantes han sido modificadas con el objetivo de mejorar la interfase hueso-implante. De hecho, desde hace más de 35 años, se acepta que la superficie de los implantes constituye uno de los factores que influyen en la cicatrización tisular del lecho implantario y como consecuencia en el fenómeno de la oseointegración ${ }^{7-9}$.

Las superficies rugosas o tratadas (ej. arenadas, grabadas) muestran un incremento en la adherencia y proliferación celular especialmente en la la actividad de los osteoblastos con una mayor producción de proteínas morfogenéticas que acelera la respuesta biológica celular ${ }^{10-11}$. Desde un punto de vista histomorfométrico, las superficies rugosas de los implantes mejoran la unión al tejido óseo del huésped. En estudios con animales, los porcentajes de contacto hueso implante de estas superficies son más elevados y su anclaje biomecánico se incrementa notablemente ${ }^{12-13}$.

El diseño macroscópico de los implantes dentales está principalmente dirigido a conseguir en la cirugía una adecuada estabilidad primaria con un torque de inserción favorable, al mantenimiento a largo plazo del hueso periimplantario y a posibilitar una adaptación a los casos clínicos diferentes en sus opciones de edentulismo parcial o total ${ }^{65}$. La macrogeometría de los implantes dentales ha sido modificada continuamente en los últimos años, con el objetivo de adaptarse y mejorar nuevos procedimientos clínicos como los implantes postextracción y la carga precoz o inmediata o la presencia de un hueso poco favorable en volumen y densidad. Actualmente los mejores resultados clínicos y de transmisión biomecánica se consiguen con una macrogeometría roscada en forma de raíz dental que logre las mejores propiedades biológicas y biomecánicas ${ }^{14}$.

La conexión prostodóncica y la plataforma del implante son determinantes para su diseño. La plataforma influye en la fase quirúrgica, y sobre la anchura biológica, pues es un área con alta concentración de estrés mecánico, e influye sobre la prótesis. La plataforma de un implante debería ser ligeramente mayor que el diámetro externo de la rosca del cuerpo del implante. De esta manera, cierra completamente la osteotomía realizada y funciona como barrera para la entrada de bacterias o tejido fibroso en la cicatrización inicial, favoreciendo la estabilidad inicial del implante ${ }^{15}$.

La estabilidad del hueso y los tejidos blandos alrededor de los implantes y pilares dentales, parece ser un elemento clave para el éxito a largo plazo del tratamiento ${ }^{16}$. En este sentido, el mantenimiento de la altura del hueso crestal juega un papel esencial en la supervivencia y el éxito a largo plazo de los implantes dentales. Los cambios de plataforma pueden ayudar a disminuir las fuerzas oclusales e incrementar la altura de la mucosa de inserción disminuyendo la inflamación de los tejidos blandos periimplantarios con una menor reabsorción ósea y mucositis ${ }^{17}$.

CONCEPTO DE PLATAFORMA REDUCIDA O PLATFORM SWITCHING

El concepto de cambio de plataforma de los implantes 
dentales, plataforma reducida o platform switching se propuso en un intento de minimizar la reabsorción ósea crestal, siendo introducido por Lazzara y Porter 18 en el año 2006, y ha sido confirmado en diversos estudios clínicos y experimentales a lo largo de los años. Las observaciones histológicas y radiográficas, sugieren que existe una dimensión biológica de los tejidos duros y blandos alrededor de los implantes dentales y se extiende apicalmente desde la interfase entre el implante y el pilar. La evidencia radiográfica del desarrollo de la dimensión biológica puede demostrarse mediante el reposicionamiento vertical del hueso crestal y la subsiguiente fijación de los tejidos blandos al implante que se produce cuando se descubre un implante, se expone al entorno oral y se colocan los componentes restauradores de diámetro equivalente ${ }^{18}$.

A lo largo de la evolución de la implantología oral, los sistemas de implantes dentales de dos piezas se habían restaurado con componentes protésicos que localizaban la interfase entre el implante y el elemento protésico acoplado en el borde exterior de la plataforma del implante. Sin embargo, cuando se introdujeron, hubo un retraso en la producción de los componentes protésicos de diámetro equivalente, que no estaban disponibles en ese momento, y muchos de los primeros implantes de 5 y $6 \mathrm{~mm}$ de ancho recibieron pilares de cicatrización de diámetro estándar $(4,1 \mathrm{~mm})$ y se restauraron con componentes protésicos de diámetro estándar $(4,1 \mathrm{~mm})^{18}$.

El seguimiento radiográfico a largo plazo de estos implantes dentales de diámetro ancho restaurados con cambio de plataforma o plataforma reducida demostró un cambio vertical menor de lo esperado en la altura del hueso crestal alrededor de estos implantes, que el que se observaba típicamente alrededor de los implantes restaurados convencionalmente con componentes protésicos de diámetros coincidentes. Esta observación radiográfica sugería que el proceso biológico postoperatorio resultante, que provocaba la pérdida de altura ósea crestal, se alteraba cuando el borde exterior de la interfase implante-pilar se reposicionaba horizontalmente hacia el interior y se alejaba del borde exterior de la plataforma del implante. En otras palabras, el concepto de cambio de plataforma se basaba en la posibilidad de alejar el espacio de unión entre el pilar protésico y el implante lejos del hombro del mismo para intentar minimizar la pérdida ósea ${ }^{18}$.

Lazzara y Porter ${ }^{18}$ descubrieron que, durante un período de 13 años de observación radiográfica periapical de implantes más anchos con pilares reducidos, se reveló una mejor preservación del hueso crestal, pero se concluyó que eran necesarias más investigaciones para probar las ventajas reales de este procedimiento clínico.

La plataforma reducida o platform switching consiste en la utilización de un pilar de menor diámetro del de la plataforma del implante (Figura 1) y se ha ido imponiendo a nivel prostodóncico con el principal objetivo de preservar el nivel de hueso crestal alrededor de los implantes dentales, con las consiguientes ventajas estéticas en el frente anterior. La preservación del hueso alrededor del implante es crucial, sobre todo en sectores anteriores ya que permitirá el soporte de los tejidos blandos manteniendo la integridad de la papila y como consecuencia, una estética mejor ${ }^{19}$.

El efecto del platform switching se produce cuando el diámetro del pilar es menor que el diámetro de la plataforma del implante, lo que resulta en un desplazamiento horizontal en la parte superior del implante que separa el hueso crestal y el tejido conectivo de la interfase implante-pilar ${ }^{20}$. La cantidad de pérdida de hueso marginal se considera un criterio de vital importancia para evaluar el resultado del tratamiento con implantes dentales y para predecir el pronóstico de la rehabilitación prostodóncica con implantes. De esta forma, la evaluación radiográfica de los cambios o modificaciones en el nivel del hueso crestal después de la restauración protésica se considera un criterio importante para evaluar los resultados clínicos del tratamiento ${ }^{21}$.

Una pérdida de hueso crestal alrededor de los implantes dentales de $1 \mathrm{~mm}$ durante el primer año, seguida de una pérdida de $0,2 \mathrm{~mm}$ en los años posteriores, ha sido considerada generalmente aceptable para implantes dentales de dos componentes 1,22. En este sentido, la pérdida de hueso crestal también puede ser considerada como el resultado de la readaptación fisiológica del posicionamiento tridimensional 
incorrecto del implante. La porción coronal del hueso puede tender a reabsorberse si se ha colocado demasiado cerca de los dientes o implantes adyacentes o de una pared vestibular residual delgada. La razón existente detrás de la reabsorción ósea fisiológica, se encuentra en el restablecimiento del ancho biológico de la zona periimplantaria ${ }^{23}$.

Sin embargo, más recientemente, diversos estudios experimentales e investigaciones clínicas han mostrado una menor pérdida marginal de hueso cuando se tienen en cuenta algunos requisitos biológicos y quirúrgicos. Algunos factores como la calidad de los tejidos periimplantarios, el trauma quirúrgico, la distancia interimplante, la presencia del microgap en la interfase pilar-implante, la posición crestal o subcrestal del implante, el macrodiseño del implante (ej. plataforma reducida), el tipo de conexión protésica y la superficie del cuello del implante son importantes en este sentido ${ }^{24}$.

Los beneficios biológicos y clínicos de la plataforma reducida se han discutido en diversos estudios que indican una reducción en la pérdida ósea marginal comparados con los implantes con plataforma convencionales. Esta minimización en los cambios óseos crestales periimplantarios incluye una mejor distribución de las cargas oclusales en la función, lo que también evita la inflamación localizada de los tejidos blandos ${ }^{25}$.

Diversos estudios histomorfomé-

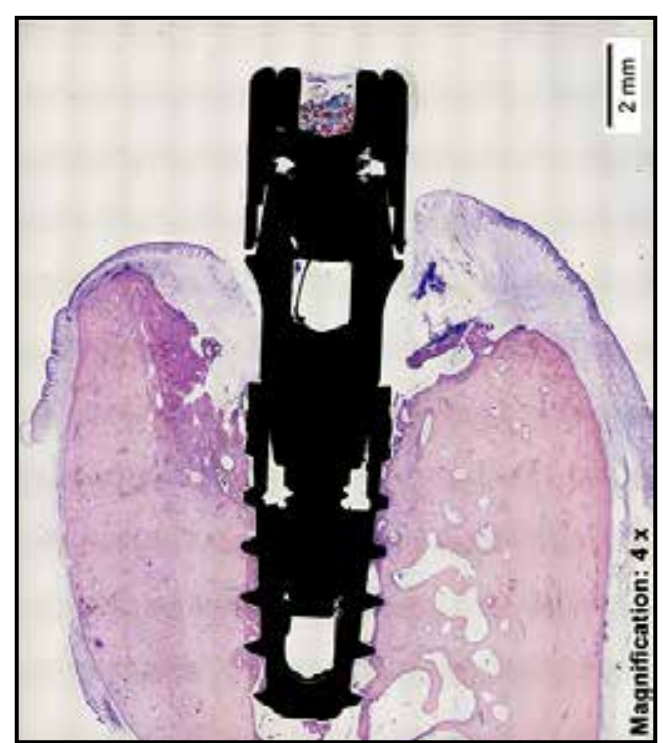

Figura 2. Imagen histológica de implante con plataforma reducida en mandíbula de perro Beagle.
Con respecto a las características macro y microscópicas de los implantes, se han descrito estudios con diversos diseños macroscópicos como en forma radicular, cónica o cilíndrica y diferentes tipología de espiras. Diferentes conexiones implante-pilar internas y externas han sido investigadas. Así mismo se han ensayados implantes con plataforma reducida con diversos tipos de tratamiento en su superficie como arenada, grabada, arenada y grabada y con revestimiento ${ }^{26}$.

\section{ESTUDIOS EXPERIMENTALES DE}

LA PLATAFORMA REDUCIDA EN ANIMALES

Para valorar la eficacia de los implantes con plataforma reducida o platform switching se han realizado estudios histológicos e histomorfométricos en animales, especialmente en perros Beagle para analizar el grado de oseointegración y el nivel de los tejidos duros y blandos periimplantarios (Figura 2) ${ }^{27-33}$.

En 2007, Becker y cols ${ }^{27}$ realizaron un estudio histológico experimental en 9 perros Beagle. Se colocaron 54 implantes de 5 $\mathrm{mm}$ de diámetro por $11 \mathrm{~mm}$ de longitud con pilares de cicatrización de 5 y $4 \mathrm{~mm}$ de diámetro y $4 \mathrm{~mm}$ de altura. Se evaluaron las muestras a los 7, 14 y 28 días tras la cicatrización. Las conclusiones del estudio fueron que en los implantes con platform switching se preservaba más la cresta alveolar $(0,5 \mathrm{~mm} \pm 0,5 \mathrm{~mm}$ en la pared vestibular y de $0,1 \mathrm{~mm}$ $\pm 0,5 \mathrm{~mm}$ en la pared lingual) tricos y de elementos finitos han mostrado el papel potencial de la plataforma reducida para limitar la reabsorción ósea periimplantaria, optimizando los espacios para los componentes biológicos, medializando el microgap implante-pilar y el infiltrado celular inflamatorio, y disminuyendo el estrés biomecánico hacia el eje central del implante ${ }^{25-26}$.

La plataforma reducida se ha utilizado en implantes con diferentes protocolos quirúrgicos como técnica sumergida o no sumergida, colocación crestal o subcrestal, inserción de implantes inmediatos después de la extracción o diferidos después de un periodo de cicatrización, con restauraciones inmediatas provisionales o definitivas diferidas ${ }^{26}$. comparada con los implantes convencionales.

Bressan y cols ${ }^{28}$ realizaron un estudio en seis perros Labrador, dónde evaluaron cómo influía en la adaptación del tejido duro periimplantario la configuración del aspecto marginal de los implantes colocados de forma inmediata en alvéolos postextracción. Los resultados del estudio demostraron histológicamente que el uso de implantes con diámetro reducido en su aspecto coronal podía contribuir a la preservación de la cresta alveolar en un nivel más coronal en comparación con los implantes de plataforma convencional. Así, el estudio confirmó la eficacia del concepto de cambio de plataforma. 
La plataforma reducida o platform switching puede también influir en conseguir una mejor estabilidad de la mucosa periimplantaria. En un estudio de experimentación animal con perros se colocaron un total de doce implantes en una sola fase quirúrgica (seis con reducción de la plataforma y seis con plataforma convencional) ${ }^{29}$. Los pilares de los implantes se desconectaron y volvieron a conectarse después de 8 , 10,12 y 14 semanas. Los animales fueron sacrificados en la semana 18. La discrepancia en el diámetro de los pilares de los implante que implica un cambio de una plataforma de implante más ancha a un diámetro de pilar más pequeño, provocaron una orientación circular de las fibras de colágeno a nivel de la plataforma del implante. Los resultados histológicos apoyaron la hipótesis de que la plataforma reducida o platform switching puede reducir la pérdida ósea crestal y servir como un factor de retención mecánica por la orientación de las fibras de colágeno ${ }^{29}$.

Otro estudio realizado en seis hembras adultas de perros Beagle dónde se colocaron un total de 48 implantes, se investigó desde el punto de vista histológico el efecto sobre los tejidos marginales periimplantarios (tejidos duros y blandos) tras la retirada repetida de los pilares con plataforma reducida y su posterior reconexión ${ }^{30}$. La conexión/desconexión de los pilares con cambio de plataforma durante la fase protésica del tratamiento con implantes no induce a la reabsorción ósea. Además, puede presentar una influencia en la unión del tejido conectivo periimplantario, que reduzca la reabsorción de los tejidos duros ${ }^{30}$.

Oscarsson y cols ${ }^{31}$ llevaron a cabo otra investigación en la que colocaron un total de 48 implantes en 6 hembras de Labrador para estudiar los tejidos blandos y duros alrededor de los implantes con plataforma reducida y con plataforma convencional, colocados en una posición crestal o subcrestal y mantenidos con o sin higiene bucal durante 5 meses. La preservación del nivel óseo fue similar independientemente de la colocación crestal o subcrestal de los implantes con cambio de plataforma hasta 5 meses después de la colocación. A pesar de ello, la colocación subcrestal de los implantes con plataforma convencional se asoció con una mayor pérdida ósea 5 meses después de la colocación en comparación con la colocación crestal. Los implantes con plataforma reducida demostraron una pérdida ósea crestal significativamente menor en comparación con los implantes de plataforma convencional ${ }^{31}$.

La posibilidad de modificar la rugosidad de la plata- forma del implante está basado en los resultados de un estudio que demuestra como el tejido óseo es capaz de crecer sobre el microgap y establecer un primer contacto hueso-implante de forma directa sobre el pilar de cicatrización en implantes insertados subcrestalmente. En esta situación, una superficie rugosa más que una superficie lisa o mecanizada puede estimular el crecimiento óseo sobre la unión o interfase implante-pilar ${ }^{32}$. En este sentido, la superficie modificada parcial o totalmente rugosa de la plataforma reducida puede ofrecer unas condiciones óptimas de cicatrización tanto en implantes inmediatos como diferidos para estimular la adhesión y proliferación de los fibroblastos y osteoblastos y conseguir una mejor respuesta tisular de los tejidos duros y blandos periimplantarios ${ }^{16}$ (Figuras).

Estos estudios realizados en perros, han sido confirmados por los resultados obtenidos en monos, donde se compararon el nivel óseo marginal de los implantes con plataforma reducida y plataforma convencional $^{33}$. La histomorfometría mostró que se había producido una reabsorción ósea alveolar tanto vertical como horizontal alrededor de los implantes convencionales, mientras que el hueso se mantenía verticalmente y se formaba hueso nuevo horizontalmente alrededor de los implantes con plataforma reducida ${ }^{33}$.

\section{EsTudios CLINICOS DE}

\section{LA PLATAFORMA REDUCIDA EN PACIENTES}

En la última década, el mantenimiento de los tejidos periimplantarios a través de la utilización de implantes con plataforma reducida o platform switching ha ido ganando un mayor reconocimiento por los profesionales de la implantologia oral y se ha ido incorporando a la práctica im-

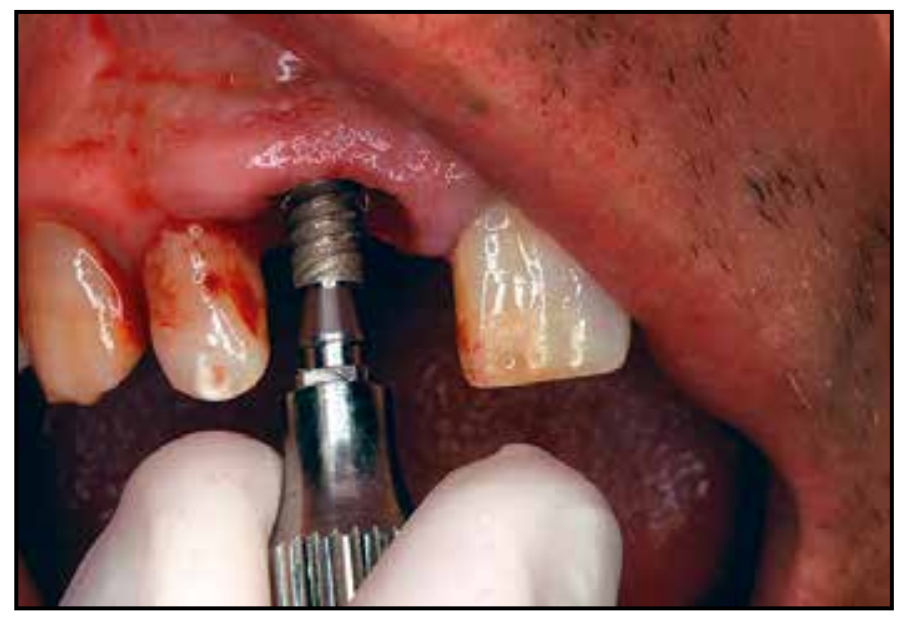

Figura 3. Inserción de implante IPX ${ }^{\circledR}$ con plataforma reducida. 
plantológica cotidiana (Figuras 3 y 4). Teniendo en cuenta el diseño del sistema de implantes dentales, el concepto de un diámetro de pilar más pequeño en relación a la plataforma para mantener y mejorar los tejidos periimplantarios ha seguido mostrando una clara evidencia de éxito clínico ${ }^{34-35}$.

En 2007, Hurzeler y cols $^{36}$ realizaron un estudio prospectivo sobre 15 pacientes en los que se colocaron implantes con platform switching en comparación a implantes convencionales. Se llevaron a cabo radiografías periapicales tras la cirugía y al año, y los resultados demostraron una menor pérdida ósea marginal en los implantes con plataforma reducida. En 2008, Capiello y cols ${ }^{37}$ realizaron un estudio clínico y radiológico dónde analizaron la pérdida ósea en implantes con platform switching en comparación con implantes convencionales. Se colocaron $131 \mathrm{im}$ plantes no sumergidos en 45 pacientes. De los 131 implantes, en 75 implantes se colocó un pilar de cicatrización $1 \mathrm{~mm}$ más estrecho que el diámetro del implante, y en 54 implantes del mismo tamaño del diámetro del implante. Se realizó un análisis previo y después de la cirugía, a las 8 y 16 semanas y a los 12 meses tras la carga. En los implantes con platform switching hubo de 0,6 a $1,2 \mathrm{~mm}$ de pérdida ósea y en los convencionales de 1,3 a 2,1 $\mathrm{mm}$ de pérdida ósea. En 2010, Fickl y cols ${ }^{38}$ llevaron a cabo un estudio similar en 10 pacientes tratados con implantes y protocolizados con platform switching. Inmediatamente después de la colocación y al año, se hizo una medición del hueso crestal sobre las superficies mesial y distal. En los implantes con platform switching la pérdida ósea fue de 0,2 $\pm 0,4 \mathrm{~mm}$ y en los convencionales de $1,2 \pm 0,3 \mathrm{~mm}$. Las conclusiones fueron que los implantes con platform switching parecen limitar la reabsorción ósea crestal y son capaces de preservar el nivel de hueso periimplantario.

Prosper y cols $^{39}$ hicieron un estudio multicéntrico en el que se colocaron 360 implantes a 60 pacientes llevando a cabo una comparación entre implantes sumergidos con pilar reducido e implantes no sumergidos. Se llevó un análisis de los datos a los 12 y 24 meses. Las conclusiones del estudio fueron que en los implantes con pilar estándar (no platform switching) se muestra más pérdida ósea que en los platform switching. La respuesta del hueso fue más homogénea en los platform switching comparados con los no platform switching.

Algunas revisiones bibliográficas publicadas en los últimos 10 años, comparando implantes restaurados con reducción de la plataforma e implantes con plataforma convencional han comparado la supervivencia de los implantes y la pérdida ósea marginal alrededor de los implantes dentales mediante estudios radiográficos con plataforma reducida con los implantes dentales de plataforma convencional ${ }^{40-43}$. También se han comparado las tasas de fracaso, así como la infección postoperatoria en implantes con plataforma reducida y con plataforma convencional ${ }^{40-43}$.

En una de las revisiones bibliográficas, los resultados indicaron que había una pérdida ósea marginal significativamente menor en los implantes con plataforma reducida que en los implantes con plataforma convencional ${ }^{40}$. Sin embargo, dichas conclusiones debían interpretarse con cautela, puesto que los periodos de seguimiento a los que fueron sometidos la mayoría de los implantes, eran relativamente cortos. Ciertas variables como el fracaso del implante o la infección postoperatoria no se analizaron debido a la falta de información ${ }^{40}$.

Romanos y cols ${ }^{41}$ realizaron a cabo otra revisión bibliográfica en la que se concluyó que la pérdida ósea alrededor de los implantes parece ser causada por varios factores; como las características cervicales del diseño del implante, el posicionamiento del implante tridimensionalmente, el concepto protésico y la conexión implante-pilar, el ancho de la cresta alveolar y la prevención de micromovimientos en la interfase implante-pilar, y no simplemente la colocación de los implantes de acuerdo con el concepto de la reducción de la plataforma ${ }^{41}$.

Se han realizado diferentes estudios en los últimos cinco años en los que los resultados mostraban una tendencia a favorecer la técnica de reducción de la plataforma para prevenir o minimizar la pérdida ósea marginal periimplantaria en comparación con la técnica de plataforma convencional ${ }^{42-}$ ${ }^{43}$. Uno de los estudios, exponía que los implantes dentales con conexiones internas mostraron una pérdida ósea marginal menor que los implantes con conexiones externas. Este hallazgo es principalmente el resultado del concepto de plataforma reducida que se encuentra con mayor frecuencia 
en los implantes con conexiones internas ${ }^{43}$.

Di Girolamo y cols ${ }^{21}$ concluyeron en su estudio que los datos relacionados con un seguimiento más prolongado se asociaron con mejores resultados en los implantes con reducción de la plataforma en comparación con los implantes con plataforma convencional.

Los resultados de un estudio realizado por Macedo y cols ${ }^{15}$, mostraron que la reducción de plataforma conserva más hueso periimplantario, estabiliza más los tejidos blandos, reduce el tamaño del microespacio que se encuentra en la conexión implante-pilar y favorece una geometría adecuada en los espacios mesiodistales edéntulos más estrechos.

Los implantes con reducción de la plataforma, pueden proporcionar un significativo efecto protector en los tejidos duros y blandos alrededor del implante en comparación con los resultados obtenidos en implantes restaurados sin reducción de la plataforma, mostrándose además resultados en los que se pone de manifiesto la estabilidad de los tejidos blandos alrededor del implante. Se ha de tener en cuenta, que la presencia de un biotipo grueso, parece ser crucial en la reducción del remodelado óseo a nivel de la cresta ${ }^{35}$.

\section{CONCLUSIONES}

La evidencia científica experimental y clínica indica que la utilización de la plataforma reducida o platform switching en la unión implante pilar mejora la unión de los tejidos duros y blandos alrededor de los implantes consiguiendo una buena estabilidad biológica y clínica que es esencial para su mantenimiento y éxito a largo plazo

\section{BIBLIOGRAFIA}

1. Albrektsson T, Zarb G, Worthington P, Eriksson AR. The long-term efficacy of currently used dental implants: a review and proposed criteria of success. Int J Oral Maxillofac Implants 1986; 1:11-25.

2. Del Fabbro M, Testori T, Kekovic V, Goker F, Tumedei M, Wang HL. Systematic review of survival rates of osseointegrated implants in fully and partially edentulous patients following immediate loading. J Clin Med 2019, 8, 2142.
3. Blanco López PP, Monsalve Guil L, Matos Garrido N, Moreno Muñoz J, Nuñez Márquez E, Velasco Ortega E. La oseointegración de implantes de titanio con diferentes superficies rugosas. Av Odontoestomatol 2018; 34: 141-149.

4. Bragança LK, Monsalve Guil L, Jiménez Guerra A, España López A, Ortiz García I, Velasco Ortega E. La biología de la oseointegración en los implantes postextraccion. Av Odontoestomatol 2018; 34:131-139.

5. Jemat A, Ghazali, MJ. Razali M, Otsuka Y. Surface modifications and their effects on titanium dental implants. BioMed Res Int 2015, 791725.

6. Nicolas-Silvente AI, Velasco-Ortega E, Ortiz-Garcia I, Monsalve-Guil L, Gil J, Jiménez-Guerra A. Influence of the titanium implant surface treatment on the surface roughness and chemical composition. Materials 2020; 13:314.

7. Carmo Filho LC, Marcello-Machado RM, Castilhos ED, del bel Cury AA, Faot F. Can implant surfaces affect implant stability during osseointegration? A randomized clinical trial. Braz Oral Res 2018; 32: 110.

8. Velasco-Ortega E, Ortiz-García I, Jiménez-Guerra J, Monsalve-Guil L, Muñoz-Guzón F, Gil FJ. Comparison between sandblasted-acid etched and oxidized titanium dental implants: In vivo study. J Mol Sci 2019; 20:13.

9. Barfeie A,Wilson J, Rees J. Implant surface characteristics and their effect on osseointegration. Br Dent J 2015; 218: 9.

10. Velasco-Ortega E, Alfonso-Rodríguez CA, Monsalve-Guil L, España-López A, Jiménez-Guerra A, Garzón I, Alaminos M, Gil FJ. Relevant aspects in the surface properties in titanium dental implants for the cellular viability. Mater Sci Eng C 2016; 64: 1-10.

11. Blatt S, Max Pabst AM, Schiegnitz E, Hosang M, Ziebart T, Walter C, Al-Nawas B, Klein MO. Early cell response of osteogenic cells on differently modified implant surfaces: Sequences of cell proliferation, adherence and differentiation. J CranioMaxilloFac Sur 2018; 46: 453-460.

12. Velasco E, Monsalve-Guil L, Jiménez A, Ortiz 
I, Moreno-Muñoz J, Nuñez-Marquez E, Pegueroles $M$, Pérez RA, Gil FJ. Importance of the roughness and residual stresses of dental implants on fatigue and osseointegration behavior. In vivo study in rabbits. J Oral Impl 2016; 42: 469-476.

13. Cho SA, Park KT. The removal torque of titanium screw inserted in rabbit tibia treated by dual acid etching. Biomaterials 2003; 24: 3611-3617.

14. Jokstad A, Sanz M, Ogawa T, Bassi F, Levin L, Wennerberg A, Romanos GE. A systematic review of the role of implant design in the rehabilitation of the edentulous maxilla. Int $\mathrm{J}$ Oral Maxillofac Implants 2016; 31:43-99.

14. Gehrke S, Bragança LK, Velasco-Ortega E, Calvo-Guirado JL. Evaluation of dimensional behavior of peri-implant tissues in implants immediately exposed or submerged in fresh extraction and healed sites: a histological study in dogs. Int J Implant Dent 2018 4:5.

15. Macedo JP, Pereira J, Vahey BR, Henriques B, Benfatti CA, Magini RS, López-López J, Souza JCM. Morse taper dental implants and platform switching: The new paradigm in oral implantology. Eur J Dent 2016;10: 148-154.

16. Montero J, Fernández-Ruiz J, Velasco-Ortega E. Effect of rough surface platforms on the mucosal attachment and the marginal bone loss of implants: A dog study. Materials 2020; 13:802.

17. Al Amri MD, Al-Johany SS, Al Baker AM, Al Rifaiy MQ, Abduljabbar TS, Al-Kheraif AA. Soft tissue changes and crestal bone loss around platform-switched implants placed at crestal and subcrestal levels: 36 -month results from a prospective split-mouth clinical trial. Clin Oral Impl Res 2017; 28:1342-1347.

18. Lazzara RJ, Porter SS. Platform switching: a new concept in implant dentistry for controlling postrestorative crestal bone levels. Int J Periodontics Restorative Dent 2006; 26:9-17.

19. Calvo Guirado JL, Ortiz Ruiz AJ, López Marí L, Delgado Ruiz R, Maté Sánchez J, Bravo González LA. Immediate maxillary restoration of single-tooth implants using platform switching for crestal bone preservation: a 12 month study. Int J Oral Maxillofac Implants 2009; 24:275-281.

20. Gargari M, Comuzzi L, Bazzato MF, Sivolella S, di Fiore A, Ceruso FM. Treatment of peri-implantitis: description of a technique of surgical 2 detoxification of the implant. A prospective clinical case series with 3-year follow-up. Oral Implantol 2015; 8:1-11.

21. Di Girolamo M, Calcaterra R, Di Gianfilippo R, Arcuri C, Baggi L. Bone level changes around platform switching and platform matching implants: a systematic review with meta-analysis. Oral Implantol 2016; 9:1-10.

22. Albrektsson T, Branemark PI, Hansson HA, Lindstrom J. Osseointegrated titanium implants. Requirements for ensuring a long-lasting, direct bone-to-implant anchorage in man. Acta Orthop Scand 1981; 52:155-170.

23. Tomasi C, Sanz M, Cecchinato D, Pjetursson B, Ferrus J, Lang NP, Lindhe J. Bone dimensional variations at implants placed in fresh extraction sockets: a multilevel multivariate analysis. Clin Oral Implants Res 2010; 21:30-36.

24. Valles $C$, Rodríguez-Ciurana X, Clementini $M$, Baglivo M, Paniagua B, Nart J. Influence of subcrestal implant placement compared with equicrestal position on the peri-implant hard and soft tissues around platform-switched implants: a systematic review and meta-analysis. Clin Oral Investig 2018; 22:555-570.

25. Pan YH, Lin HK, Lin JCY, Yung-Szu Hsu YS, Wu YF, Salamanca E, Chang WJ. Evaluation of the peri-implant bone level around platform-switched dental implants: a retrospective 3-year radiographic study. Int $\mathrm{J}$ Environ Res Public Health 2019; 16:2570.

26. Annibali S, Bignozzi I, Cristalli MP, Graziani F, La Monaca G, Polimeni A. Peri-implant marginal bone level: a systematic review and meta-analysis of studies comparing platform switching versus conventionally restored implants. J Clin Periodontol 2012; 39: 1097-1113.

27. Becker J, Ferrari D, Herten M, Kirsch A, Schaer A, Schwarz F. Influence of platform switching on crestal bone changes at non-submerged ti- 
tanium implants: a histomorphometrical study in dogs. J Clin Periodontol 2007; 34:10891096.

28. Bressan E, Lang NP, Corazza B, Ricci S, Almagro Urrutia Z, Botticelli D. The platform switching concept revisited. An experimental study in dogs. Clin Oral Implants Res 2014; 25:12001206.

29. Rodríguez X, Vela X, Calvo-Guirado JL, Nart J, Stappert CF. Effect of platform switching on collagen fiber orientation and bone resorption around dental implants: a preliminary histologic animal study. Int $\mathrm{J}$ Oral Maxillofac Implants 2012; 27:1116-1122.

30. Alves C, Muñoz F, Cantalapiedra A, Ramos I, Neves M, Blanco J. Marginal bone and soft tissue behavior following platform switching abutment connection/disconnection. A dog model study. Clin Oral Impl Res 2015; 26:983-991.

31. Oskarsson M, Otsuki M, Welander M, Abrahamson I. Peri囚implant tissue healing at implants with different designs and placement protocols: An experimental study in dogs. Clin Oral Impl Res 2018; 29:873-880.

32. Weng D, Nagata MJH, Bell M, Bosco AF, de Melo LGN, Richter, EJ. Influence of microgap location and configuration on the periimplant bone morphology in submerged implants. An experimental study in dogs. Clin Oral Impl Res 2008; 19:1141-1147.

33. Makigusa K, Toda I, Yasuda K, Ehara D, Suwa F. Effects of platform switching on crestal bone around implants: a histomorphometric study in monkeys. Int J Periodontics Restorative Dent 2014; 34:35-41.

34. Santiago JF, Batista VE, Verri FR, Honório HM, de Mello CC, Almeida DA, Pellizzer EP. Platform-switching implants and bone preservation: a systematic review and meta-analysis. Int J Oral Maxillofac Surg 2016; 45:332-345.

35. Hsu Y T, Lin G H, Wang H L. Effects of platform-switching on peri-implant soft and hard tissue outcomes: A systematic review and meta-analysis. Int $\mathrm{J}$ Oral Maxillofac Implants 2017; 32: 9-24.
36. Hürzeler M, Fickl S, Zuhr O, Wachtel HC. Peri-implant bone level around implants with platform-switched abutments: preliminary data from a prospective study. J Oral Maxillofac Surg 2007; 65:33-39.

37. Cappiello M, Luongo R, Di lorio D, Bugea C, Cocchetto R, Celletti R. Evaluation of peri-implant bone loss around platform-switched implants. Int $\mathrm{J}$ Periodontics Restorative Dent. 2008; 28:347-355.

38. Fickl S, Zuhr O, Stein JM, Hürzeler MB. Peri-implant bone level around implants with platform-switched abutments. Int J Oral Maxillofac Implants 2010; 25:577-581.

39. Prosper L, Redaelli S, Pasi M, Zarone F, Radaelli G, Gherlone EF. A randomized prospective multicenter trial evaluating the platform-switching technique for the prevention of postrestorative crestal bone loss. Int J Oral Maxillofac Implants 2009; 24:299-308.

40. Chrcanovic BR, Albrektsson T, Wennerberg A. Platform switch and dental implants: A meta-analysis. J Dent 2015; 43:629-646.

41. Romanos GE, Javed F. Platform switching minimises crestal bone loss around dental implants: truth or myth? J Oral Rehabil 2014; 41:700-708.

42. Strietzel FP, Neumann K, Hertel M. Impact of platform switching on marginal peri-implant bone-level changes. A systematic review and meta-analysis. Clin Oral Implants Res. 2015; 26:342-358.

43. Medeiros RA, Pellizzer EP, Vechiato Filho AJ, Dos Santos DM, da Silva EV, Goiato MC. Evaluation of marginal bone loss of dental implants with internal or external connections and its association with other variables: A systematic review. J Prosthet Dent 2016; 116:501-506.

\section{AUTOR DE CORRESPONDENCIA:}

Prof. Eugenio Velasco Ortega

Facultad de Odontología

C/ Avicena s/n 41009 Sevilla

Email: evelasco@us.es

Tfno: 954481132 